\title{
Measurements of the hadronic cross sections with the CMD-3 and SND detectors at the VEPP-2000 $e^{+} e^{-}$ collider
}

M.N.Achasov ${ }^{a b}$, R.R.Akhmetshin ${ }^{a}$, A.V.Anisenkov ${ }^{a}$, V.M.Aulchenko ${ }^{a b}$, V.S.Banzarov ${ }^{a}$, L.M.Barkov $^{a}$, A.Yu.Barnyakov ${ }^{a}$, N.S.Bashtovoy ${ }^{a}$, K.I.Beloborodov $^{a}{ }$, A.V.Berdyugin $^{a b}$, D.E.Berkaev $^{a b}$, A.G.Bogdanchikov ${ }^{a}$, A.E.Bondar ${ }^{a b}$, A.A.Botov ${ }^{a}$, A.V.Bragin $^{a}$, T.V.Dimova ${ }^{a}$, V.P.Druzhinin ${ }^{a b}$, S.I.Eidelman ${ }^{a b}$, D.A.Epifanov ${ }^{a f}$, L.B.Epshteyn $^{a c}$, A.L.Erofeev ${ }^{a}$, G.V.Fedotovich ${ }^{a b}$, S.E.Gayazov ${ }^{a b}$, V.B.Golubev $^{a b}$, A.A.Grebenuk $^{a b}$, K.A.Grevtsov ${ }^{a b}$, D.N.Grigoriev ${ }^{a b c}$, E.M.Gromov ${ }^{a}$, F.V.Ignatov ${ }^{* a}$, L.V.Kardapoltsev $^{a b}$, S.V.Karpov ${ }^{a}$, V.F.Kazanin ${ }^{a b}$, A.G.Kharlamov ${ }^{a b}$, B.I.Khazin ${ }^{a b}$, A.N.Kirpotin ${ }^{a}$, I.A.Koop ${ }^{a b}$, A.A.Korol ${ }^{a b}$, S.V.Koshuba ${ }^{a}$, O.A.Kovalenko ${ }^{a b}$, D.P.Kovrizhin ${ }^{a}$, A.N.Kozyrev ${ }^{a}$, E.A.Kozyrev ${ }^{a b}$, P.P.Krokovny $^{a b}$, A.S.Kupich ${ }^{a}$, A.E.Kuzmenko ${ }^{a c}$, A.S.Kuzmin ${ }^{a}$, I.B.Logashenko ${ }^{a b}$, P.A.Lukin ${ }^{a b}$, A.P.Lysenko ${ }^{a}$, K.A.Martin $^{a c}$, K.Yu.Mikhailov ${ }^{a b}$, A.E.Obrazovsky ${ }^{a}$, V.S.Okhapkin ${ }^{a}$, E.V.Pakhtusova ${ }^{a}$, E.A.Perevedentsev ${ }^{a b}$, Yu.N.Pestov ${ }^{a}$, A.S.Popov ${ }^{a b}$, Yu.S.Popov ${ }^{a}$, G.P.Razuvaev ${ }^{a b}$, Yu.A.Rogovsky ${ }^{a}$, A.L.Romanov ${ }^{a}$, A.A.Ruban ${ }^{a}$, N.M.Ryskulov $^{a}$, A.E.Ryzhenenkov ${ }^{a b}$, S.I.Serednyakov ${ }^{a b}$, P.Yu.Shatunov ${ }^{a}$, Yu.M.Shatunov ${ }^{a}$, V.E.Shebalin ${ }^{a}$, D.N.Shemyakin $^{a b}{ }$ D.A.Shtol $^{a}$, B.A.Shwartz ${ }^{a b}$, D.B.Shwartz $^{a b}$, A.L.Sibidanov $^{a d}$, Z.K.Silagadze $^{a b}$, E.P.Solodov ${ }^{a b}$, I.K.Surin ${ }^{a}$, A.A.Talyshev ${ }^{a b}$, V.M.Titov $^{a}$, A.V.Vasiljev ${ }^{a}$, A.I.Vorobiov $^{a}$, Yu.V.Yudin ${ }^{a}$, I.M.Zemlyansky ${ }^{a}$, Yu.M.Zharinov ${ }^{a}$

${ }^{a}$ Budker Institute of Nuclear Physics, SB RAS, Novosibirsk, 630090, Russia

${ }^{b}$ Novosibirsk State University, Novosibirsk, 630090, Russia

${ }^{c}$ Novosibirsk State Technical University, Novosibirsk, 630092, Russia

${ }^{d}$ University of Sydney, Falkiner High Energy Physics, NSW 2006, Sydney, Australia

${ }^{e}$ Brookhaven National Laboratory, P.O. Box 5000 Upton, NY 11973-5000, USA

${ }^{f}$ University of Tokyo, Department of Physics, 7-3-1 Hongo Bunkyo-ku Tokyo, 113-0033, Japan

E-mail: ignatov@inp.nsk.su

Regular data taking with the CMD-3 and SND detectors at the electron-positron collider VEPP2000 is under way since December 2010. The collected data sample corresponds to about 60 inverse picobarns of integrated luminosity per detector in the energy range from 0.32 up to $2 \mathrm{GeV}$. Preliminary analysis of various hadronic cross sections has been performed.

The European Physical Society Conference on High Energy Physics -EPS-HEP2013

18-24 July, 2013

Stockholm, Sweden

* Speaker. 


\section{Introduction}

The electron-positron collider VEPP-2000 [1,2] has been operating at Budker Institute of Nuclear Physics since 2010. The collider is designed to provide luminosity up to $10^{32} \mathrm{~cm}^{-2} \mathrm{~s}^{-1}$ at the maximum center-of-mass energy $\sqrt{s}=2 \mathrm{GeV}$. At present two detectors, CMD-3 $[3,4]$ and SND [5], are installed in the interaction regions of the collider. In 2010 both experiments started data taking. The current integrated luminosity is about $2 \times 60 \mathrm{pb}^{-1}$. The physics program [6] includes high precision measurements of the $e^{+} e^{-} \rightarrow$ hadrons cross sections in the wide energy range up to $2 \mathrm{GeV}$, studies of known and searches for new vector mesons, studies of $n \bar{n}$ and $p \bar{p}$ production cross sections near threshold and searches for exotic hadrons. It requires detectors with high efficiency for multiparticle events and good energy and angular resolution for charged particles as well as for photons.

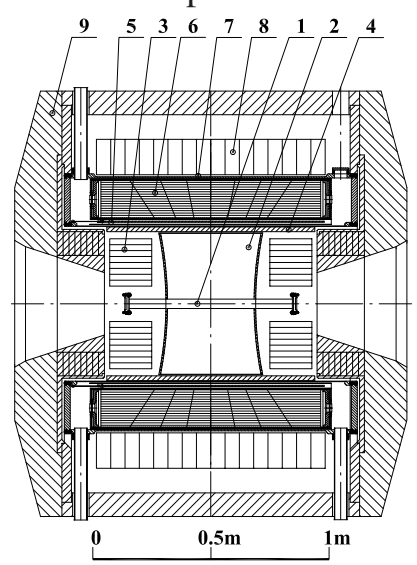

Figure 1: CMD-3 detector: 1 - beam pipe, 2 - drift chamber, 3 - BGO calorimeter, 4 - Z-chamber, 5 SC solenoid $\left(0.13 X_{0}, 13 \mathrm{kGs}\right), 6$ - LXe calorimeter, 7 - TOF system, 8 - CsI electromagnetic calorimeter, 9 - yoke, not shown muon range system

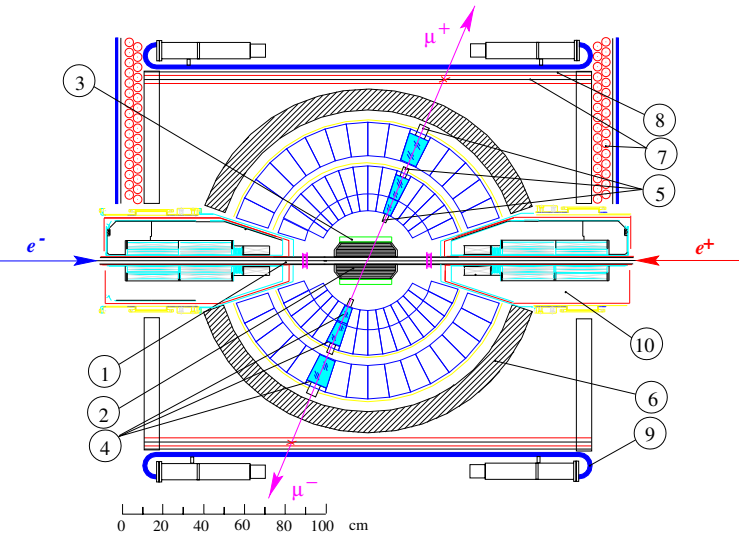

Figure 2: SND detector, section along the beams: 1 beam pipe, 2 - tracking system, 3 - aerogel Cherenkov counter, 4 - NaI(Tl) crystals, 5 - phototriodes, 6 iron absorber, 7 - streamer tubes, 8 - iron plates, 9 scintillation counters, 10 - solenoid of VEPP-2000.

CMD-3 (Cryogenic Magnetic Detector) is a general purpose detector, see Fig. 1. Coordinates, angles and momenta of charged particles are measured by the cylindrical drift chamber with a hexagonal cell for uniform reconstruction of tracks.

The calorimetry is performed with the endcap BGO calorimeter and the barrel calorimeter. Barrel calorimeter placed outside of the superconducting solenoid providing 1.3 T magnetic field consists of two systems: inner ionisation Liquid Xenon calorimeter which is comprised by the CsI scintillation calorimeter. The total thickness of the barrel calorimeter is about $13.5 X_{0}$. The LXe calorimeter has seven layers with strip readout which give information about a shower profile and are also able to measure coordinates of photons with high accuracy about millimeter precision.

SND (Spherical Neutral Detector) is optimized to detect neutral final states, see Fig. 2. Its main part is a highly segmented spherical three-layer $\mathrm{NaI}(\mathrm{Tl})$ calorimeter with 560 individual crystals per layer and $90 \%$ solid angle coverage. There is a tracking system around the collider beam pipe, based on a nine-layer drift chamber and one-layer proportional chamber with cathode strip readout. Outside the calorimeter a muon detector consisting of proportional tubes and scintillation counters 


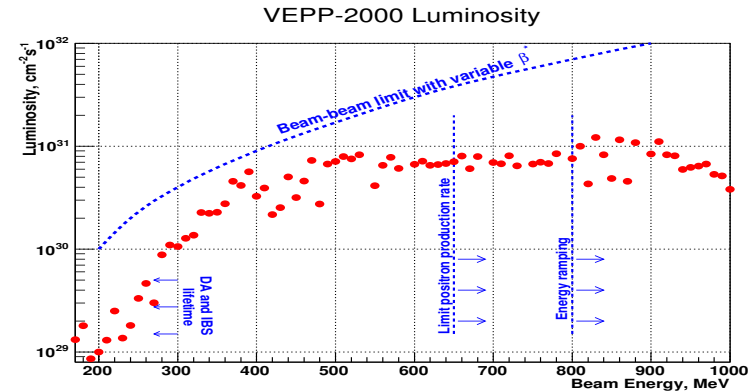

Figure 3: Luminosity delivered by VEPP-2000 averaged over top $10 \%$ runs. The current limitations are lack of positrons and beam lifetime at low energy

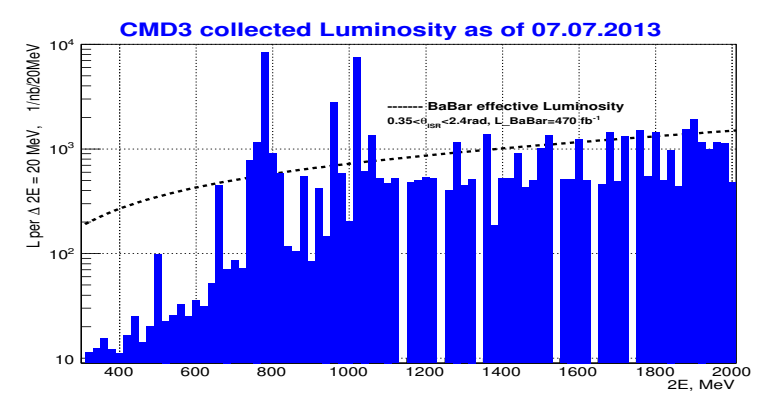

Figure 4: Luminosity collected by CMD-3. The effective ISR luminosity of BaBar (the last competitor experiment) is shown for comparison

is placed. An aerogel Cherenkov counter located between the drift chamber and the calorimeter is used for particle identification.

The $10^{31} \mathrm{~cm}^{-2} \mathrm{~s}^{-1}$ luminosity was reached by the VEPP-2000 collider (Fig. 3). The already collected integrated luminosity is $60 \mathrm{pb}^{-1}$ per detector, with about $34.5 \mathrm{pb}^{-1}$ above the $\phi$ energy, 8.3 and $8.4 \mathrm{pb}^{-1}$ at the $\omega$ and $\phi$ resonances, respectively, and $9.4 \mathrm{pb}^{-1}$ from a scan below the $\phi$ (Fig. 4). The luminosity at high energy is currently limited by a deficit of positrons and maximum energy of the booster ( $825 \mathrm{MeV}$ now), and after upgrade of the accelerator complex it will gain a factor of ten. The new positron injection facility and achieved operational experience will also improve the situation at low energy.

\section{Pion formfactor}

The dominant contribution to $R(s)$ in the energy range $\sqrt{s}<1 \mathrm{GeV}$ comes from the $e^{+} e^{-} \rightarrow \pi^{+} \pi^{-}$mode. At CMD-2 this process was measured in the energy range from $0.37 \mathrm{GeV}$ to $1.38 \mathrm{GeV}[7,8,9,10]$. The $0.6-0.8 \%$ systematic uncertainty of this measurement was achieved for $\sqrt{s}<1 \mathrm{GeV}$. For energies above $1 \mathrm{GeV}$ it varies from $1.2 \%$ to $4.2 \%$. SND measured the $e^{+} e^{-} \rightarrow \pi^{+} \pi^{-}$cross section in the energy range $0.39-0.97 \mathrm{GeV}$ with the systematic uncertainty of $1.3 \%$ [11]. The first energy scan below $1 \mathrm{GeV}$ was performed at VEPP-2000 during the season of 2013. A very preliminary result on formfactor measurement is shown in

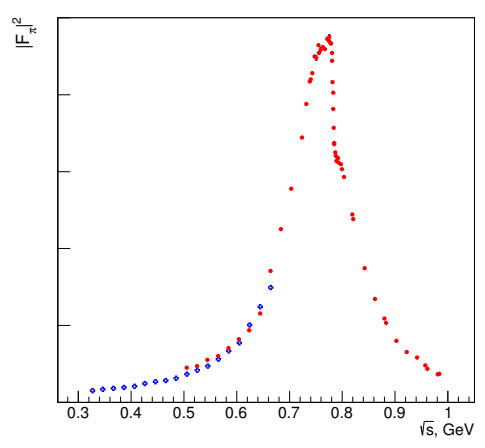

Figure 5: Prelimenary results on $F_{\pi}^{2}$ from CMD-3. Filled circles - separation done from the calorimeter information, open crosses - from particle momentum

Fig. 5. The already collected statistics is higher than that in the previous CMD-2 experiment and at the level or better than in the BaBar and KLOE experiments (Fig. 6).

The goal of the CMD-3 experiment is to reduce a systematic uncertainty on this channel to $0.35 \%$. The geometry of this process allows a clean selection of $e^{+} e^{-}, \mu^{+} \mu^{-}, \pi^{+} \pi^{-}$collinear events to be performed practically without physical background. These final states can be separated using either the information about energy deposition in the calorimeter or that about particle momenta in the drift chamber at energy $E_{\text {beam }} \lesssim 330 \mathrm{MeV}$. These two methods overlap in the energy range $E_{\text {beam }} \approx 250 \div 330 \mathrm{MeV}$ and crosscheck each other, allowing a systematic error of event separation at the level of $0.2 \%$ to be kept. In the CMD-3 detector, determination of the fiducial 


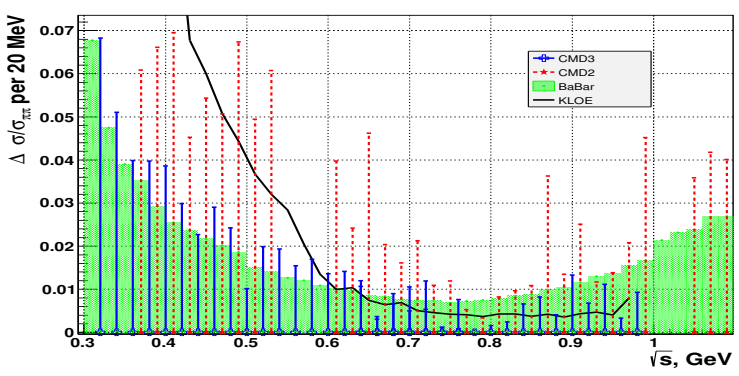

Figure 6: Statistical precision of $\left|F_{\pi}\right|^{2}$ from the CMD3 data in comparison with CMD-2, BaBar and KLOE results volume could be made independently with the LXe and Z-chamber subsystems. It allows an efficient monitoring of detector operation stability during data taking. An addition of other methods to crosscheck a Z scale measurement (radiography of detector elements from conversion of particles on it, momentum versus polar angle correlation and so on) will allow to keep a systematic uncertainty from this source at the $0.1 \%$ level. Measurement of beam energy by Compton backscattering of the laser photons with precision $\sigma_{E}<50 \mathrm{keV}$ [12] will keep a systematic uncertainty from this source below $0.1 \%$. Another important source of systematics is theoretical precision of radiative corrections [13]. Additional studies like crosschecks of different calculation approaches and further proof from comparison with experimental data are necessary in this field. We expect that this uncertainty can be reduced to $0.1 \%$.

One of the tests in this analysis is a measurement of the $e^{+} e^{-} \rightarrow \mu^{+} \mu^{-}$cross section at low energy, where separation was performed using momentum information. Preliminary results of this test are consistent with the QED prediction with an overall precision of $0.5 \%$ as shown in Fig. 7.

\section{3. $K^{+} K^{-} \pi^{+} \pi^{-}$}

The important part of $e^{+} e^{-} \rightarrow$ hadrons production above the $\phi$ energy comes from channels with kaons. Fig. 8 shows the preliminary CMD-3 results on the $e^{+} e^{-} \rightarrow K^{+} K^{-} \pi^{+} \pi^{-}$cross section in comparison with other experiments. Good agreement with BaBar data is seen whereas DM2 data are systematically lower. This channel has rich dynamics with many intermediate states like: $K^{*}(1270) K \rightarrow K^{*}(892) K \pi, K^{*}(1400) K \rightarrow K^{*}(892) K \pi, K^{*}(1270) K \rightarrow \rho K K, K^{*}(892) K^{*}(892)$, $\phi \pi \pi$. Fig. 9 show dominance of the $K^{*}(892)$ intermediate state in the $K \pi$ invariant mass, and presence of the $\rho$ in the $\pi^{+} \pi^{-}$combination. For calculations of the hadronic contributions to $(g-2)_{\mu}$ and $\alpha_{Q E D}\left(M_{Z}\right)$ it is necessary to measure all possible $K K \pi, K K \pi \pi$ cross sections with neutral and charged kaons in the final state.

\section{Multipion production}

The multipion production $\left(e^{+} e^{-} \rightarrow 3 \pi, \ldots, 6 \pi\right)$ has rich dynamics with many possible intermediate states. The precision of the cross sections for these channels is dominated by a model uncertainty. High statistics, which will be obtained at VEPP-2000, will allow to study dynamics with much better accuracy and reduce this source of systematics.

The total cross section of the process $e^{+} e^{-} \rightarrow 3\left(\pi^{+} \pi^{-}\right)$has been measured using $22 \mathrm{pb}^{-1}$ of integrated luminosity collected with the CMD-3 detector in the $\sqrt{s}=1.5-2.0 \mathrm{GeV}$ energy range [14]. 


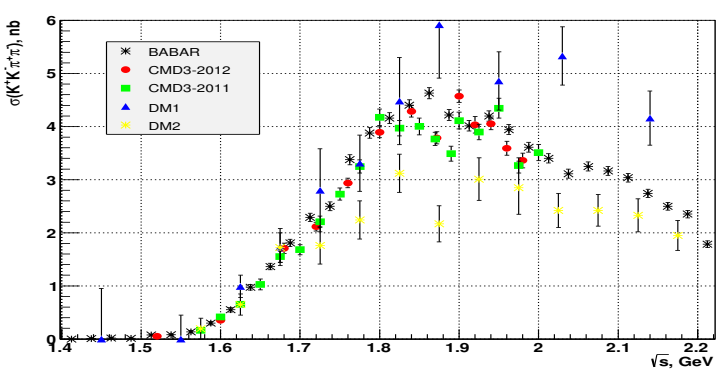

Figure 8: Preliminary result on the $e^{+} e^{-} \rightarrow$ $K^{+} K^{-} \pi^{+} \pi^{-}$cross section with CMD-3

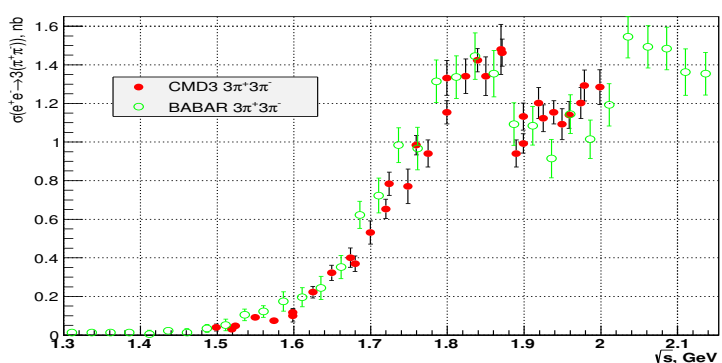

Figure 10: Measurement of the $e^{+} e^{-} \rightarrow 3\left(\pi^{+} \pi^{-}\right)$ cross section at CMD-3
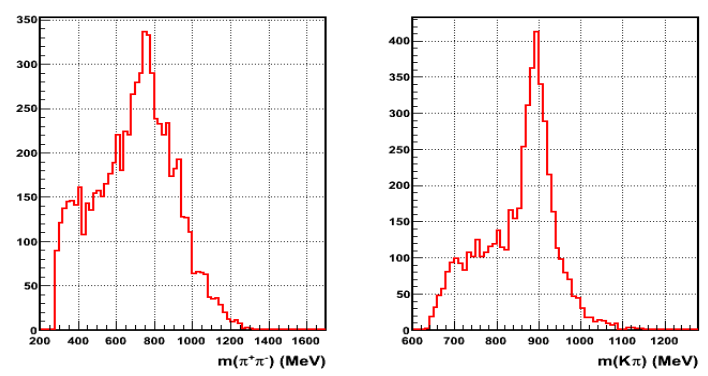

Figure 9: Invariant mass of the $\pi^{+} \pi^{-}$(left), and $K^{ \pm} \pi^{\mp}$ pair (right)

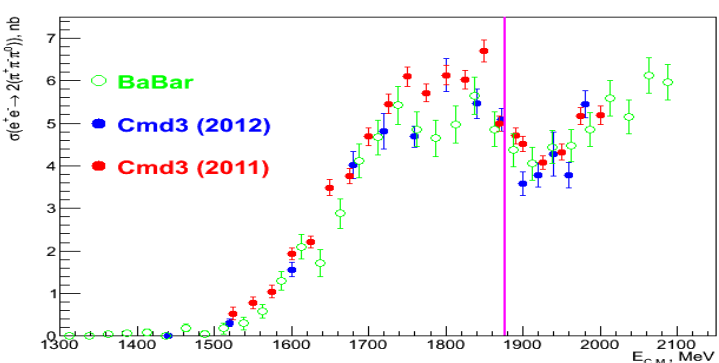

Figure 11: Measurement of the $e^{+} e^{-} \rightarrow 2\left(\pi^{+} \pi^{-} \pi^{0}\right)$ cross section at CMD-3

The measured cross section is in good agreement with all previous experiments. The ratio of the five and six track events were used to estimate the model-dependent uncertainty in the acceptance calculation. From this study it was concluded that the observed production mechanism can be described by the production of one $\rho$ (770) with four remaining pions in the S-wave distributed according to phase space. It was also observed that the production dynamics change in the $1700-1900 \mathrm{MeV}$ c.m. energy range and demand a further study. Detailed analysis of the production dynamics will be performed in the combined analysis of the processes $e^{+} e^{-} \rightarrow 3\left(\pi^{+} \pi^{-}\right)$and $e^{+} e^{-} \rightarrow 2\left(\pi^{+} \pi^{-} \pi^{0}\right)$. The preliminary results on $2\left(\pi^{+} \pi^{-} \pi^{0}\right)$ are shown in Fig. 11. A sharp dip in the $6 \pi$ cross section is seen near $p \bar{p}$ threshold (it is worth noting that the value of the jump in the $6 \pi$ production cross section is roughly equal to a sum of $p \bar{p}$ and $n \bar{n}$ cross sections). It will be interesting to see a quantitative theoretical description of this effect.

Results from SND on the $e^{+} e^{-} \rightarrow \omega \pi^{0} \rightarrow \pi^{0} \pi^{0} \gamma$ cross section in the energy range of $1.05-2.00 \mathrm{GeV}$ have already been published (Fig. 12) [15]. It is the most accurate measurement of this cross section between 1.4 and $2.0 \mathrm{GeV}$. SND data agree with the earlier measurements performed at the VEPP-2M collider with the SND [16] and CMD2 [17] detectors below $1.4 \mathrm{GeV}$. Significant disagreement with DM2 data [18] is observed in the energy range 1.3$2.0 \mathrm{GeV}$. As shown by SND, data on the $e^{+} e^{-} \rightarrow \omega \pi^{0}$ cross section are well described by the VMD model with three $\rho$ like states: $\rho(770), \rho^{\prime}$ and $\rho^{\prime \prime}$. However, the full data set on

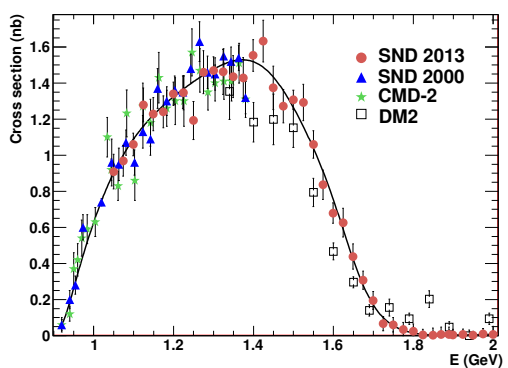

Figure 12: The $e^{+} e^{-} \rightarrow \omega \pi^{0} \rightarrow$ $\pi^{0} \pi^{0} \gamma$ cross section measurement 


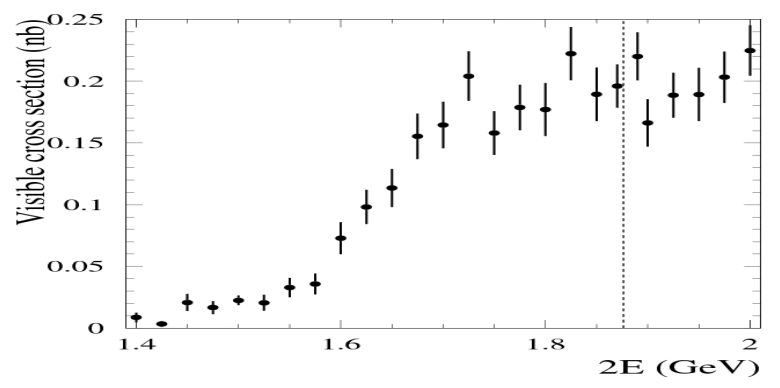

Figure 13: The $e^{+} e^{-} \rightarrow \pi^{+} \pi^{-} \pi^{0} \pi^{0} \pi^{0} \pi^{0} \rightarrow \pi^{+} \pi^{-} 8 \gamma$ cross section from SND

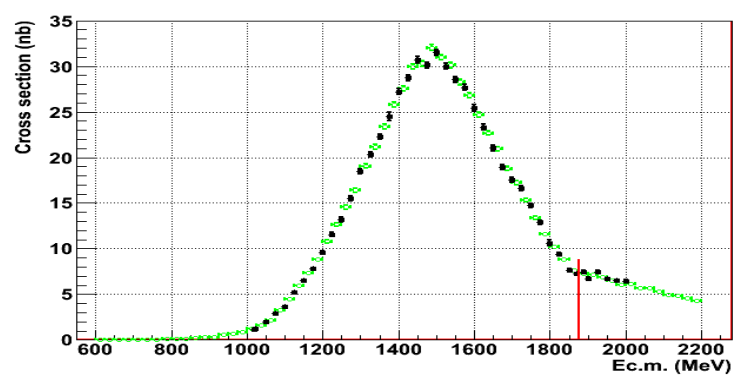

Figure 15: The $e^{+} e^{-} \rightarrow \pi^{+} \pi^{-} \pi^{+} \pi^{-}$cross section from CMD-3

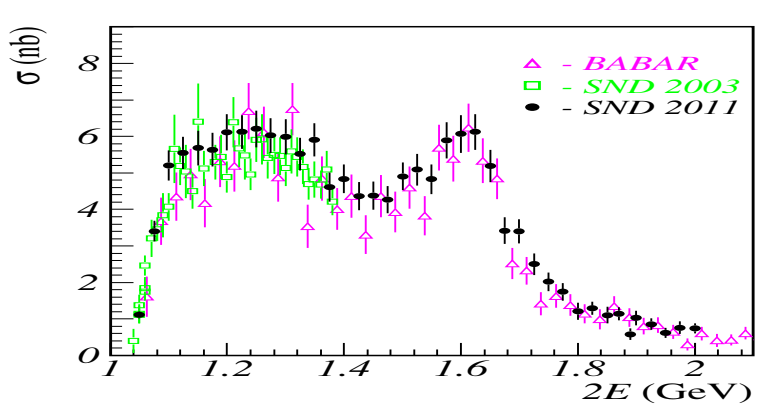

Figure 14: The $e^{+} e^{-} \rightarrow \pi^{+} \pi^{-} \pi^{0}$ cross section from SND

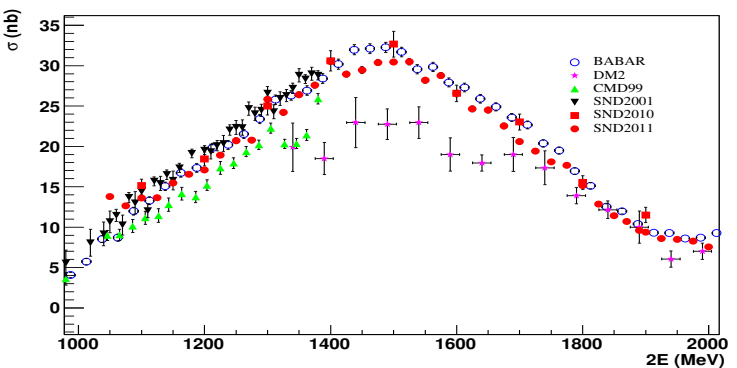

Figure 16: The $e^{+} e^{-} \rightarrow \pi^{+} \pi^{-} \pi^{0} \pi^{0}$ cross section from SND

the $\gamma^{*} \rightarrow \omega \pi^{0}$ transition form factor including data from both $e^{+} e^{-}$annihilation and $\omega$ decays, in particular, $\omega \rightarrow \pi^{0} \mu^{+} \mu^{-}$[19], cannot be described by such a simple model.

The visible cross section $e^{+} e^{-} \rightarrow \pi^{+} \pi^{-} \pi^{0} \pi^{0} \pi^{0} \pi^{0} \rightarrow \pi^{+} \pi^{-} 8 \gamma$ is shown in Fig. 13. This channel was not measured before, and in $R(s)$ is currently accounted for by isospin relations. It is important to measure all unseen cross sections and reduce uncertainties from approximate isospin relations.

In Fig. 14 preliminary results on the $e^{+} e^{-} \rightarrow \pi^{+} \pi^{-} \pi^{0}$ cross section are shown. "Plenty of statistics was collected during 2013 run for a measurement of this cross section also in the $\omega$ region. The best previous results in the $\omega$ region were obtained by CMD-2 (publication of 2000 [20]) with small systematics (1.3\%) but low statistics, and by SND, which had good statistics but worse systematics $(3.4 \%)$.

The preliminary results on $e^{+} e^{-} \rightarrow 4 \pi$ from the CMD-3 and SND detectors are shown in Figs. 15 and 16 . The $2\left(\pi^{+} \pi^{-}\right)$measurement by CMD-3 has a statistical error at the level of $1-2 \%$ per point and is in good agreement with previous measurements. The cross section of the $\pi^{+} \pi^{-} 2 \pi^{0}$ channel at energies above the $\phi$ meson is known much worse. At this moment the only published data set for the energy range above $1.4 \mathrm{GeV}$ comes from more than 30 -year-old experiments at DCI and ADONE.

\section{Nucleons near threshold}

The energy range of the VEPP-2000 collider allows to measure nucleon formfactors near threshold. Currently about $6 \mathrm{pb}^{-1}$ from $p \bar{p}$ threshold to $2 \mathrm{GeV}$ were collected. Figures 17,18 


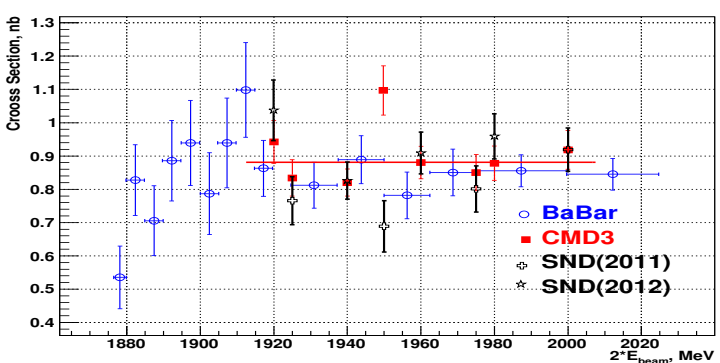

Figure 17: The $e^{+} e^{-} \rightarrow p \bar{p}$ cross section from CMD3 and SND



Figure 18: The $e^{+} e^{-} \rightarrow n \bar{n}$ cross section from SND

show the preliminary results on cross sections of nucleon pair production. The results on the $n \bar{n}$ and $p \bar{p}$ cross sections agree with those from FENICE and BaBar, respectively. It will be possible to distinguish the electrical and magnetic parts by measuring the $\left|G_{e} / G_{m}\right|$ ratio with statistical precision about $5 \%$ after a planned data sample of $1 \mathrm{fb}^{-1}$ will be collected.

\section{Conclusions}

VEPP-2000 accelerator successfully operates with a goal to get $\sim 1 \mathrm{fb}^{-1}$ in 5-10 years and provide new precise results on the hadron production. The CMD-3 and SND detectors were upgraded, with significantly improved performance and monitoring capabilities of different detector subsystems. Data analysis is in progress, the already collected data sample allows the same or better statistical precision on cross sections than previously to be achieved. First publications on data analysis already appeared $\left(6 \pi, \omega \pi^{0}\right)$. A new positron injection complex will be commissioned during an upcoming shutdown for about 1.5 year. The luminosity will be increased by a factor of 10 up to $10^{32} \mathrm{~cm}^{-2} \mathrm{~s}^{-1}$ at $2 E=2 \mathrm{GeV}$.

We thank the VEPP-2000 team for excellent machine operation.

This work is supported in part by the RFBR grants 11-02-00112-a, 11-02-00328-a, 12-0200065-a, 12-02-01032-a, 12-02-01250-a. 12-02-31498-mol-a, 12-02-31499-mol-a, 12-02-31501mol-a, 13-02-00215-a, and the DFG grant GZ: HA 1457/7-2.

\section{References}

[1] Y. .M. Shatunov et al., Conf. Proc. C 0006262 (2000) 439

[2] D. Berkaev et al., Nucl. Phys. Proc. Suppl. 225-227 (2012) 303

[3] V. M. Aulchenko et al., BUDKER-INP-2001-45

[4] B. Khazin,Nucl. Phys. Proc. Suppl. 181-182 (2008) 376

[5] M. N. Achasov et al.,Nucl. Instrum. Meth. A 598 (2009) 31

[6] S. Eidelman,Nucl. Phys. Proc. Suppl. 162 (2006) 323

[7] R. R. Akhmetshin et al., Phys. Lett. B 578 (2004) 285

[8] V. M. Aulchenko et al., JETP Lett. 82 (2005) 743
[9] R. R. Akhmetshin et al., JETP Lett. 84 (2006) 413

[10] R. R. Akhmetshin et al., Phys. Lett. B 648 (2007) 28

[11] M. N. Achasov et al., J. Exp. Theor. Phys. 103 (2006) 380

[12] E. V. Abakumova et al.,Phys. Rev. Lett. 110 (2013) 140402

[13] S. Actis et al., Eur. Phys. J. C 66 (2010) 585

[14] R. R. Akhmetshin et al.,Phys. Lett. B 723 (2013) 82

[15] M. N. Achasov et al.,Phys. Rev. D 88, 054013 (2013)

[16] M. N. Achasov et al., Phys. Lett. B 486 (2000) 29

[17] R. R. Akhmetshin et al.,Phys. Lett. B 562 (2003) 173

[18] D. Bisello et al.,Nucl. Phys. Proc. Suppl. 21 (1991) 111

[19] R. Arnaldi et al.,Phys. Lett. B 677 (2009) 260

[20] R. R. Akhmetshin et al.,Phys. Lett. B 476 (2000) 33 